\title{
Determinação espectrofotométrica do bromidrato de fenoterol em preparações farmacêuticas
}

\author{
Maria Lucilia Motinha Zamuner ${ }^{* 1}$, Regina Celli Espires Carrion', João Fernandes Magalhães² \\ 'Departamento de Farmácia e Farmacologia, Universidade Estadual de Maringá, ²Departamento de Farmácia, \\ Faculdade de Ciências Farmacêuticas, Universidade de São Paulo
}

*Correspondência:

M. L. M. Zamuner

Departamento de Farmácia e

Farmacologia

Universidade Estadual de Maringá

Av. Colombo, 5790

87020-900 - Maringá - PR, Brasil.

E-mail: mlmzamuner@uem.br
Um método espectrofotométrico simples foi desenvolvido para a determinação do bromidrato de fenoterol (BF) em comprimidos, gotas e xarope, como princípio ativo único e associado ao ibuprofeno. O método se baseia na reação de acoplamento oxidativo do BF com o 3-metil-2-benzotiazolinona hidrazona (MBTH), na presença de sulfato cérico, como agente oxidante. A mistura de BF, MBTH e sulfato cérico, em meio ácido, produz um composto colorido (vermelho castanho) com máximo de absorção a $475 \mathrm{~nm}$. A curva de calibração foi linear num intervalo de concentração de 3,0 a 12,0 $\mu \mathrm{g} / \mathrm{mL}$, com coeficiente de correlação linear de 0,9998. Os parâmetros experimentais que afetam o desenvolvimento e a estabilidade do produto colorido foram cuidadosamente estudados e otimizados. O método foi aplicado em amostras comerciais e simuladas, obtendo-se coeficientes de variação entre $0,25 \%$ a $0,82 \%$ e médias de recuperação do padrão que variaram de $98 \%$ a $102 \%$. O método proposto mostrou-se exato, preciso, linear e não é passível de interferência de excipientes, para as formas farmacêuticas comprimidos e gotas. Não houve interferência do ibuprofeno que consta de uma das formulações analisadas, associado ao BF. Quanto ao xarope, houve interferência do veículo sugerindo reações de seus componentes com o MBTH.
Unitermos

- Bromidrato de fenoterol/ determinação

- 3-metil-2-benzotiazolinona hidrazona

- Formulações farmacêuticas/ análise

- Espectrofotometria/ análise de medicamentos

\section{INTRODUÇÃO}

O fenoterol é uma mistura do $(R)$-1-(3,5dihidroxifenil)-2-[(R)-4-hidroxi- $\alpha$-metilfenetilamino) etanol (Figura 1) e seu enantiômero. Sua fórmula molecular como bromidrato: $\mathrm{C}_{17} \mathrm{H}_{21} \mathrm{NO}_{4}, \mathrm{HBr}$ e peso molecular 384,3, estão descritos na Farmacopéia Britânica (BP, 2007). É um agonista adrenérgico $\beta_{2}$-seletivo, usado como broncodilatador e tocolítico. As especialidades farmacêuticas comercializadas no Brasil, que contém o BF como princípio ativo, estão sob a forma de comprimidos, solução oral (gotas), xarope e aerossol dosificador para inalação. É comercializado, também, em associação com o ibuprofeno, um analgésico e antiinflamatório, derivado dos 
ácidos arilalcanóicos, sob a forma de comprimidos e com o brometo de ipratrópio, um anticolinérgico, sob a forma de aerossol dosificador.

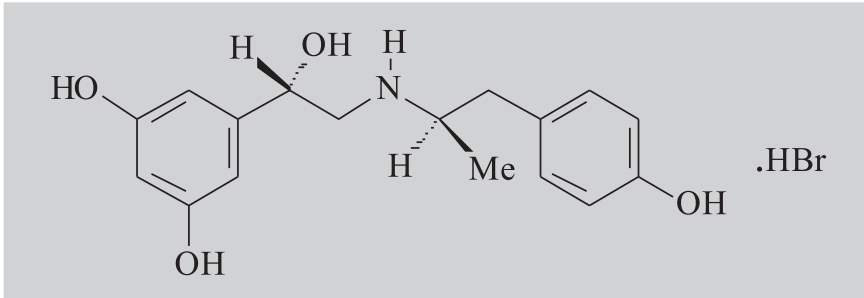

FIGURA 1 - Estrutura química do bromidrato de fenoterol

Alguns métodos analíticos foram descritos para o BF como único fármaco ou associado a outros agonistas $\beta_{2}$ adrenérgicos. Entre estes métodos citamos a determinação volumétrica indireta (método de Volhard), por precipitação do brometo com excesso de nitrato de prata, descrita para a matéria-prima na Farmacopéia Britânica (BP, 2007). No entanto, a determinação coulométrica de alguns antiasmáticos, incluindo o BF foi descrita por Nikolic, Arsenijevec e Bogavac (1993). Belal, Al-Malaq e Al-Majed (2000) descreveram o doseamento voltamétrico do fenoterol associado a um vasodilatador - a isoxsuprina, em medicamentos e fluídos biológicos. A cromatografia líquida de alta eficiência para a determinação do BF em plasma humano após derivatização com 9-cloroformil-carbazole foi descrita por Kramer e Blaschke (2001) e Meineke et al. (2002). Jacobson e Peterson (1994) descreveram a determinação do fenoterol e outros agentes $\beta_{2}$-agonistas adrenérgicos por cromatografia líquida de alta eficiência, em nebulizadores. A detecção na urina por cromatografia gasosa acoplada a espectrometria de massas de vários agonistas adrenérgicos, incluindo o fenoterol, foi descrita por Damasceno et al. (2002). El-Shabrawy et al. (2003) e Beyene, Staden e Stefan (2004) relataram a determinação espectrofotométrica do BF após derivatização, produzindo compostos coloridos medidos na região do visível.

São poucos os trabalhos descritos na literatura para análise do BF por espectrofotometria e por ser uma técnica simples, rápida e sensível pode ser uma opção econômica para farmácias de manipulação e indústrias farmacêuticas. Em uma das preparações farmacêuticas comercializadas no Brasil, o BF está associado ao ibuprofeno, um analgésico e antiinflamatório. Como ambos absorvem na mesma região do ultravioleta, este trabalho tem como objetivo propor um método de determinação espectrofotométrica no visível para o $\mathrm{BF}$, isolado ou quando associado ao ibuprofeno. O método se fundamenta na reação de acoplamento oxidativo do fenoterol com o reagente colorimétrico conhecido como 3-metil-2-benzotiazolinona hidrazona (MBTH). Este agente cromogênico reage com compostos fenólicos (Geeta, Baggi, 1988), aminas aromáticas e alifáticas (El-Emam et al., 2003), aldeídos (Rao et al., 1988), e outros, na presença de um reagente oxidante como o cloreto férrico, sulfato ou nitrato cérico em meio adequado (ácido, básico ou neutro). O produto colorido obtido do acoplamento oxidativo pode ser medido espectrofotometricamente, o que constitui a base da determinação quantitativa de diversos fármacos (Rahman, Khan, Azmi, 2004; El-Emam et al., 2003; Sultan, 2002; Prasad et al., 2002; Nagaraja et al., 2002).

\section{MATERIAL}

\section{Padrões e reagentes}

O BF e o ibuprofeno utilizados como padrões foram gentilmente doados por uma indústria farmacêutica. $\mathrm{O}$ reagente 3-metil-2-benzotiazolinona hidrazona (MBTH), o sulfato cérico e o ácido sulfúrico são, respectivamente, de procedência Sigma e Merck, de grau analítico. Na padronização do método e aplicação em amostras comerciais e simuladas, as soluções padrão de BF e das amostras foram preparadas nas concentrações de $50,0 \mathrm{mg} / \mathrm{mL}$ e $35,0 \mathrm{mg} / \mathrm{mL}$ em ácido sulfúrico $1 \mathrm{M}$. O MBTH $0,5 \mathrm{mg} / \mathrm{mL}$ e o sulfato cérico $1,0 \mathrm{mg} / \mathrm{mL}$ foram preparados em ácido sulfúrico $0,5 \mathrm{M}$, sendo estas as condições de melhores resultados espectrofotométricos.

\section{Equipamentos}

Foram utilizados: balança analítica marca MettlerToledo, modelo AB204, espectrofotômetro da marca Varian, modelo Cary 1E, UV-Visível, com cubetas de quartzo de 1,0 cm e impressora, potenciômetro Gehaka, modelo PG 2000, banho-maria de 6 bocas e vidrarias calibradas.

\section{AMOSTRAS}

Ao preparar as amostras simuladas de comprimidos foram utilizados: lactose, celulose microcristalina, amido, estearato de magnésio, aerosil, polivinilpirrolidona (PVP), glicolato de amido sódico (explotab) e talco, todos de grau farmacêutico. $\mathrm{Na}$ amostra simulada $\mathrm{D}$, foi empregado, ainda, o ibuprofeno como fármaco associado. Para as amostras simuladas líquidas foram utilizados água destilada, xarope simples e benzoato de sódio. As amostras simuladas isentas de ativo (placebos) reproduziram as mesmas formulações. A Tabela I apresenta as amostras utilizadas nos ensaios. 
TABELA I - Amostras comerciais, simuladas, e suas formas farmacêuticas utilizadas neste trabalho

\begin{tabular}{lcc}
\hline Amostra $^{(a)}$ & Fármaco & Forma farmacêutica \\
\hline Amostra A (comercial) & BF & Comprimidos, $2,5 \mathrm{mg}$ \\
Amostra B (simulada) & BF & Comprimidos, 2,5 mg \\
Amostra C (comercial) & BF e Ibuprofeno & Comprimidos, $1,5 \mathrm{mg} \mathrm{e} 200 \mathrm{mg}$ \\
Amostra D (simulada) & BF e Ibuprofeno & Comprimidos, $1,5 \mathrm{mg} \mathrm{e} 200 \mathrm{mg}$ \\
Amostra E (comercial) & BF & Gotas, $5,0 \mathrm{mg} / \mathrm{mL}$ \\
Amostra F (simulada) & BF & Gotas, $5,0 \mathrm{mg} / \mathrm{mL}$ \\
Amostra G (comercial) & BF & Xarope, $0,5 \mathrm{mg} / \mathrm{mL}$ \\
Amostra H (simulada) & BF & Xarope, $0,5 \mathrm{mg} / \mathrm{mL}$ \\
\hline
\end{tabular}

(a) As amostras comerciais foram adquiridas em farmácias comerciais.

\section{MÉTODOS}

\section{Estabelecimento das condições ideais para a reação de acoplamento oxidativo entre o BF e o MBTH}

Inicialmente, o BF foi testado quanto à reatividade com o MBTH. Os fatores que influenciam a reatividade e que, portanto modificam as características espectrofotométricas como a acidez, tempo de reação, temperatura e concentração dos reagentes, foram estudados (Esquema 1).

\section{Preparo das soluções padrão de BF $50,0 \mu \mathrm{g} / \mathrm{mL}$ e $35,0 \mu \mathrm{g} / \mathrm{mL}$}

Do padrão de BF foram pesados exatamente $100,0 \mathrm{mg}$ e transferidos para balão volumétrico de $100 \mathrm{~mL}$. Adicionaram-se cerca de $10 \mathrm{~mL}$ de ácido sulfúrico $1 \mathrm{M}$ para dissolução e completou-se o volume com o mesmo ácido. Desta solução transferiu-se uma alíquota de 5,0 mL para balão volumétrico de $100 \mathrm{~mL}$ e ajustou-se o volume com ácido sulfúrico $1 \mathrm{M}$. A solução obtida contém $50,0 \mu \mathrm{g} / \mathrm{mL}$ de BF. Para o preparo da solução padrão de BF $35,0 \mu \mathrm{g} / \mathrm{mL}$,

ESQUEMA 1 - Estudo da reação entre o BF e o MBTH, na presença de sulfato cérico como agente oxidante. Avaliação da influência da acidez, concentração dos reagentes e tempo de reação

\begin{tabular}{cc}
\hline Experimento A & Experimento B \\
\hline $10 \mathrm{~mL}$ de solução aquosa de BF $50 \mu \mathrm{g} / \mathrm{mL}$ & $10 \mathrm{~mL}$ de solução de BF $50 \mu \mathrm{g} / \mathrm{mL}$ em \\
$\mathrm{H}_{2} \mathrm{SO}_{4}(0,1 \mathrm{M}$ a $1,5 \mathrm{M})$
\end{tabular}


foi adotado o mesmo procedimento, considerando-se a concentração final a ser obtida.

\section{Preparo das soluções de amostras comerciais e simuladas de BF $50,0 \mu \mathrm{g} / \mathrm{mL}$ e $35,0 \mu \mathrm{g} / \mathrm{mL}$}

Determinou-se o peso médio de uma amostra de 20 comprimidos, que foram pulverizados e homogeneizados. Para amostras simuladas, os pós correspondendo a $20 \mathrm{com}$ primidos foram pesados e misturados cuidadosamente em gral. Pesou-se o equivalente a $10,0 \mathrm{mg}$ e 7,0 mg de BF e transferiu-se para dois balões volumétricos de $200 \mathrm{~mL}$. Adicionaram-se $100 \mathrm{~mL}$ de ácido sulfúrico $1 \mathrm{M}$ em cada balão, que foram agitados por 10 minutos em ultra-som. Ajustou-se o volume de ambos com o mesmo ácido e filtrou-se cada balão rejeitando-se os primeiros $10 \mathrm{~mL}$. As soluções obtidas apresentaram, respectivamente, $50 \mu \mathrm{g} / \mathrm{mL}$ e $35 \mu \mathrm{g} / \mathrm{mL}$ de BF. Para a solução oral (gotas) e xarope, comerciais e simuladas foi adotado o mesmo procedimento, considerando-se a concentração inicial das amostras e final a ser obtida.

\section{Padronização do método}

\section{Obtenção do espectro de absorção}

Estabelecidas as condições do ensaio (acidez, tempo de reação, temperatura e concentração do MBTH e do sulfato cérico) foi determinado o espectro de absorção, transferindo-se alíquotas de $10 \mathrm{~mL}$ da solução padrão de BF $35,0 \mu \mathrm{g} / \mathrm{mL}$ para três balões volumétricos de $50 \mathrm{~mL}, 10 \mathrm{~mL}$ de ácido sulfúrico $1 \mathrm{M}, 5 \mathrm{~mL}$ de MBTH $0,5 \%$ e $5 \mathrm{~mL}$ de sulfato cérico $1,0 \%$. Para obtenção do branco foi adicionado ao balão volumétrico $20 \mathrm{~mL}$ de ácido sulfúrico $1 \mathrm{M}$, $5 \mathrm{~mL}$ de MBTH $0,5 \%$ e $5 \mathrm{~mL}$ de sulfato cérico $1,0 \%$. Os volumes de todos os balões foram ajustados com água destilada após 20 minutos de repouso. As leituras das absorvâncias foram efetuadas após 10 minutos, contra o branco, no intervalo de 300 a $700 \mathrm{~nm}$, utilizando-se cubetas de quartzo de $1 \mathrm{~cm}$.

\section{Construção da curva de Ringbom}

Da solução padrão de BF $50,0 \mu \mathrm{g} / \mathrm{mL}$ foram transferidas alíquotas variáveis entre 1,0 a $20,0 \mathrm{~mL}$ para balões volumétricos de $50 \mathrm{~mL}$. Os volumes de todas as soluções foram ajustados para $20 \mathrm{~mL}$ com ácido sulfúrico $1 \mathrm{M}$. Em todos os balões foram adicionados $5 \mathrm{~mL}$ de MBTH $0,5 \%$ e $5 \mathrm{~mL}$ de sulfato cérico $1,0 \%$. Os balões foram mantidos em repouso por 20 minutos e seus volumes ajustados com água destilada, obtendo-se soluções com intervalo de concentração de 1,0 a 20,0 $\mu \mathrm{g} / \mathrm{mL}$ de BF. As leituras das absorvâncias foram determinadas após 10 minutos de repouso a $475 \mathrm{~nm}$, contra o branco. Com os dados obtidos foi construído o gráfico da curva de Ringbom.

\section{Construção da curva de calibração}

Foram preparadas soluções no intervalo de 3,0 a $12,0 \mu \mathrm{g} / \mathrm{mL}$ de BF numa série de 10 balões volumétricos de $50 \mathrm{~mL}$. O ensaio foi conduzido nas mesmas condições da curva de Ringbom. A relação entre absorvância e a concentração foi utilizada para a construção do gráfico da curva de calibração.

\section{Validação do método analítico}

O método analítico foi validado segundo critérios estabelecidos na Farmacopéia Americana 29a edição (USP, 2006) e no "Guia para validação de métodos analíticos e bioanalíticos" da Agência Nacional de Vigilância Sanitária - ANVISA (Brasil, 2003). Foram priorizados os seguintes parâmetros: precisão, exatidão, linearidade e especificidade.

\section{Precisão e exatidão}

A precisão e a exatidão foram determinadas realizando-se dez determinações espectrofotométricas de cada solução de amostras comerciais e simuladas contra triplicata da solução padrão. Os resultados obtidos foram submetidos à análise estatística pela técnica dos mínimos quadrados, considerando-se as médias experimentais comparando-as com as médias teóricas. A precisão foi expressa pelo cálculo do desvio padrão e em função do coeficiente de variação. O teste de recuperação do padrão, descrito por Garfield (1994), foi efetuado para comprovar a exatidão do método e consistiu em adicionar às amostras quantidades conhecidas de padrão da substância em estudo e aplicação do método proposto.

\section{Linearidade}

A linearidade do método foi determinada após construção de uma reta, considerando o intervalo de concentração de 6,0 a $10,0 \mu \mathrm{g} / \mathrm{mL}$ a partir do placebo da amostra B. Além da avaliação visual gráfica, os resultados foram submetidos à análise estatística .

\section{Especificidade}

Com a finalidade de verificar a possível interferência dos demais componentes das fórmulas no método e determinar sua especificidade, prepararam-se amostras simuladas isentas de BF que foram submetidas ao método proposto.

\section{Precisão intraensaio e interensaio}

$\mathrm{Na}$ avaliação da precisão intra-ensaio foram realizadas dez determinações (em triplicata) com a amostra co- 
mercial A (comprimidos de BF) e as amostras simuladas B (comprimidos de BF), D (comprimidos de BF e Ibuprofeno) e F (gotas de BF) na concentraçao de 7,0 $\mu \mathrm{g} / \mathrm{mL}$. As análises foram realizadas durante o dia, pelo mesmo analista e mesma instrumentação. Para a avaliação da precisão interensaio foram realizadas 10 determinações (em triplicata), com as mesmas amostras na mesma concentração, porém em dias diferentes e por analistas diferentes durante cinco dias. O método foi desenvolvido conforme descrito no "Guia para validação de métodos analíticos e bioanalíticos" da ANVISA (Brasil, 2003) e os resultados foram submetidos à análise estatística.

\section{RESULTADOS E DISCUSSÃO}

No estudo das propriedades espectrofotométricas do produto colorido formado considerando-se o método proposto, algumas variáveis foram cuidadosamente estudadas e otimizadas, conforme visto no Esquema 1. Para a avaliação da interferência da acidez, a solução padrão e as soluções amostras foram preparadas em água e em diferentes concentrações de ácido sulfúrico $(0,1$ a $1,5 \mathrm{M})$. O melhor resultado obtido na absorção a $475 \mathrm{~nm}$ foi a utilização de ácido sulfúrico $1 \mathrm{M}$ no preparo destas soluções (padrão e amostras). Para a determinação da melhor concentração do MBTH e do sulfato cérico, diferentes concentrações entre $0,1 \mathrm{mg} / \mathrm{mL}$ e $2,0 \mathrm{mg} / \mathrm{mL}$, de ambos, foram ensaiadas. Os reagentes também foram testados quando preparados em água destilada ou em ácido sulfúrico a $0,5 \mathrm{M}$ e a $1 \mathrm{M}$. Os resultados demonstraram o máximo de absorção e estabilidade do produto colorido quando da adição de $5 \mathrm{~mL}$ de MBTH $0,5 \%$ e $5 \mathrm{~mL}$ de sulfato cérico $1,0 \%$, preparados em ácido sulfúrico $0,5 \mathrm{M}$. Em relação ao melhor tempo para desenvolvimento e estabilidade do produto colorido, foram estabelecidos repousos de 0 a 40 minutos antes e após os balões volumétricos serem ajustados com água destilada ou com ácido sulfúrico $0,5 \mathrm{M}$ ou $1 \mathrm{M}$, ficando estabelecido como condição ideal um repouso de vinte minutos antes e dez minutos após os ajustes de volumes com água destilada. Estas condições se aproximam daquelas já descritas na literatura por El-Emam et al. (2003) para a reação do propranolol com o MBTH e nitrato cérico amoniacal. Para avaliar a interferência de variações de temperatura, o ensaio foi conduzido à temperatura ambiente $\left( \pm 25^{\circ} \mathrm{C}\right)$ e nas temperaturas de $30{ }^{\circ} \mathrm{C}, 40{ }^{\circ} \mathrm{C}, 50{ }^{\circ} \mathrm{C}$, $60^{\circ} \mathrm{C} \mathrm{e} 70^{\circ} \mathrm{C}$. Os resultados demonstraram que não há interferência da temperatura, se o ensaio for realizado abaixo de $40{ }^{\circ} \mathrm{C}$, tornando o método exeqüível à temperatura ambiente. Estabelecidas as condições experimentais o espectro de absorção do produto colorido foi obtido (Figura 2), na qual fica evidente o máximo de absorção a $475 \mathrm{~nm}$.

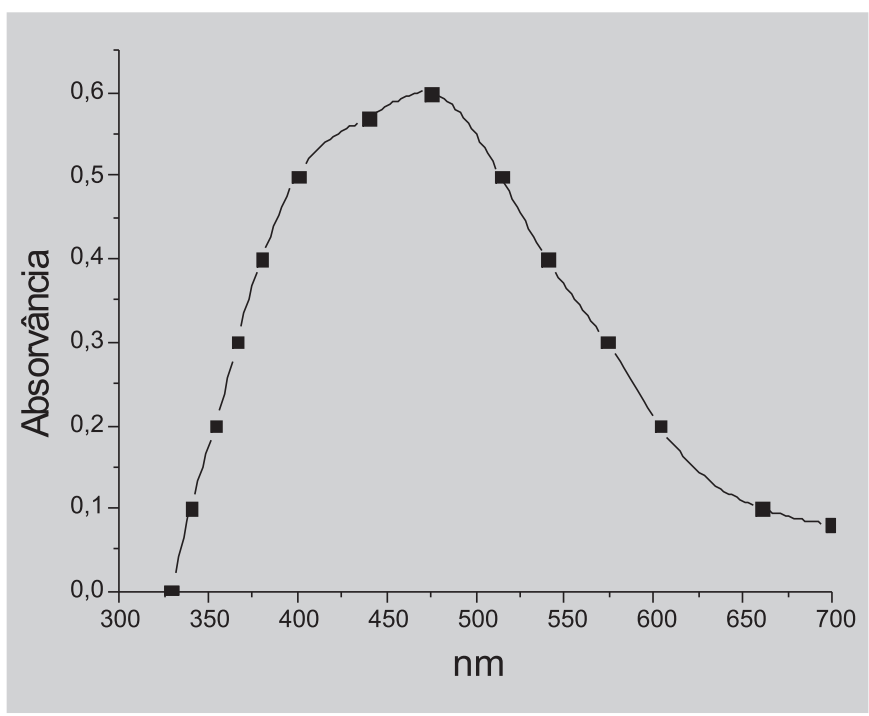

FIGURA 2 - Espectro de absorção do produto colorido. BF $7,0 \mu \mathrm{g} / \mathrm{mL}$, MBTH $0,5 \mathrm{mg} / \mathrm{mL}$ e sulfato cérico $1,0 \mathrm{mg} / \mathrm{mL}$.

Para confirmação dos resultados obtidos a partir dos espectros de absorção foi construída a curva de Ringbom através do Programa Microcal Origin 6.0. O estudo foi realizado em uma larga faixa de concentração de BF $(1,0$ a $20,0 \mu \mathrm{g} / \mathrm{mL}$ ), na qual o traçado sigmóide característico da curva de Ringbom pode ser observado na Figura 3.

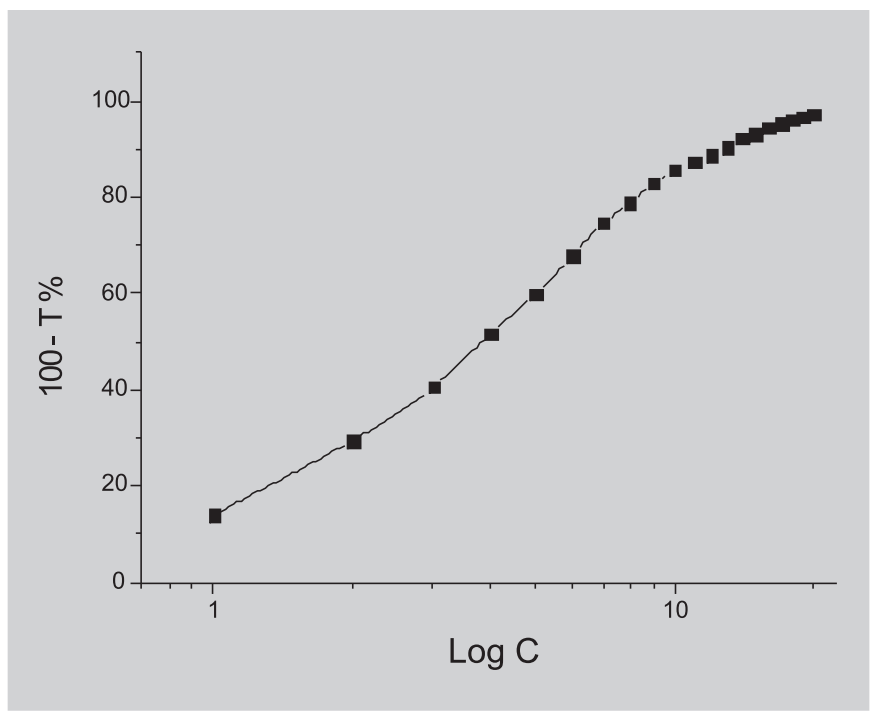

FIGURA 3 - Curva de Ringbom obtida a partir da solução de bromidrato de fenoterol nas concentrações de 1,0 a $20,0 \mu \mathrm{g} / \mathrm{mL}$, determinadas a $475 \mathrm{~nm}$, contra branco.

A porção linear da curva de Ringbom permitiu a construção da curva de calibração no intervalo de concentração de 3,0 a 12,0 $\mu \mathrm{g} / \mathrm{mL}$ de BF. A curva de calibração construída através do Programa Microcal Origin 6.0, foi igual a $\mathrm{y}=-0,00611+0,08227 \mathrm{x}$, com coeficiente de correlação (r) igual a 0,9998, (a) não significativo e erro pa- 
drão da estimativa relativo $\left(\mathrm{S}_{\mathrm{er}}\right)$ igual a $0,86 \%$. Diante destes resultados, adotou-se como parâmetro analítico ideal, a concentração de $7,0 \mu \mathrm{g} / \mathrm{mL}$ de $\mathrm{BF}$ correspondente à absorvância próxima a 0,6 , valor este que atende as recomendações apresentadas por Vogel (2002).

Considerando que a avaliação da especificidade é importante para métodos analíticos que não exigem etapa prévia de separação e, por definição, este atributo avalia a possibilidade de interferência de excipientes/impurezas no desempenho do método, as Figuras 4, 5 e 6 mostram os resultados obtidos na análise de placebos, segundo o método proposto. Observamos na Figura 4, que os excipientes e veículos presentes em comprimidos e gotas não interferem na determinação de BF. Também a presença do ibuprofeno como princípio ativo associado ao fenoterol (amostra D), não modificou o espectro de absorção de acordo com a Figura 5, comprovando a especificidade do método proposto. Entretanto, na avaliação da especificidade do método com as amostras simuladas de xarope (amostra He placebo da amostra $\mathrm{H}$ ), observou-se interferência do placebo conforme apresentado na Figura 6, sugerindo possíveis reações dos componentes do xarope com o MBTH. A amostra comercial de xarope (amostra $G$ ) também foi avaliada segundo o método proposto, porém os resultados não foram apresentados considerando que a absorção a $475 \mathrm{~nm}$ foi aproximadamente $50 \%$ maior do que aquela obtida com o padrão de BF nas mesmas condições e concentração, sugerindo forte interferência dos componentes da fórmula.

Após análises das amostras simuladas e comerciais e avaliação estatística dos resultados encontrados, apresentados na Tabela II, conclui-se que o método proposto é

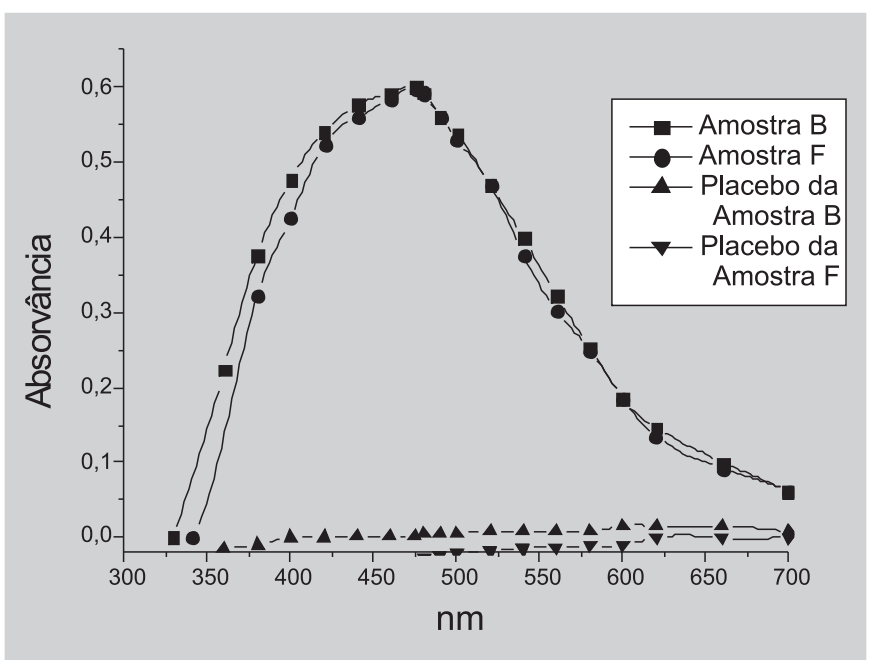

FIGURA 4 - Avaliação da influência dos placebos das amostras simuladas B (comprimidos) e F (gotas) no espectro de absorção do produto colorido. Concentração de leitura: $7,0 \mu \mathrm{g} / \mathrm{mL}$ de BF.

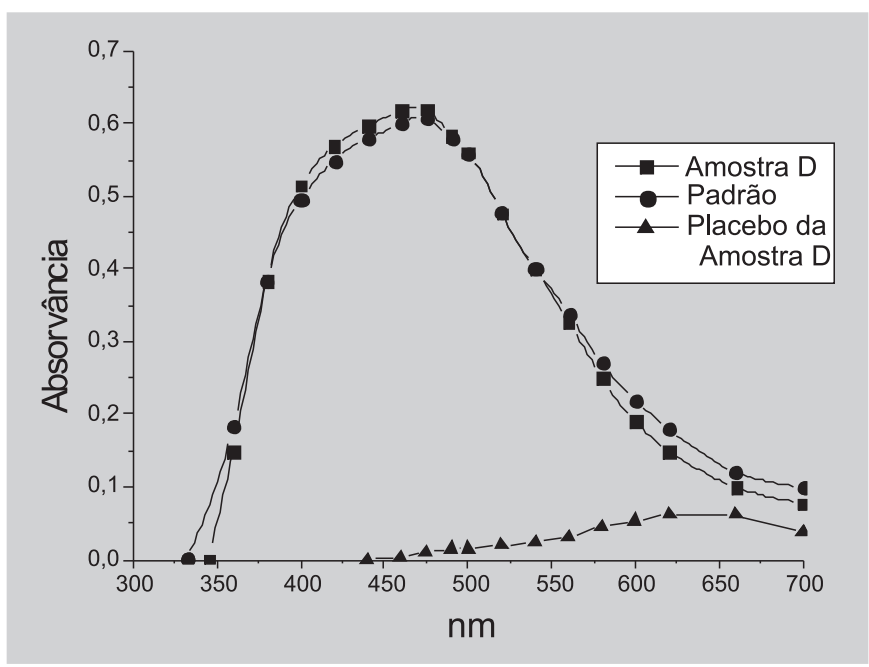

FIGURA 5 - Avaliação da influência do ibuprofeno e do placebo da amostra simulada D (comprimidos de BF e ibuprofeno) no espectro de absorção do produto colorido. Concentração de leitura do padrão e da amostra: 7,0 $\mu \mathrm{g} / \mathrm{mL}$ de BF.

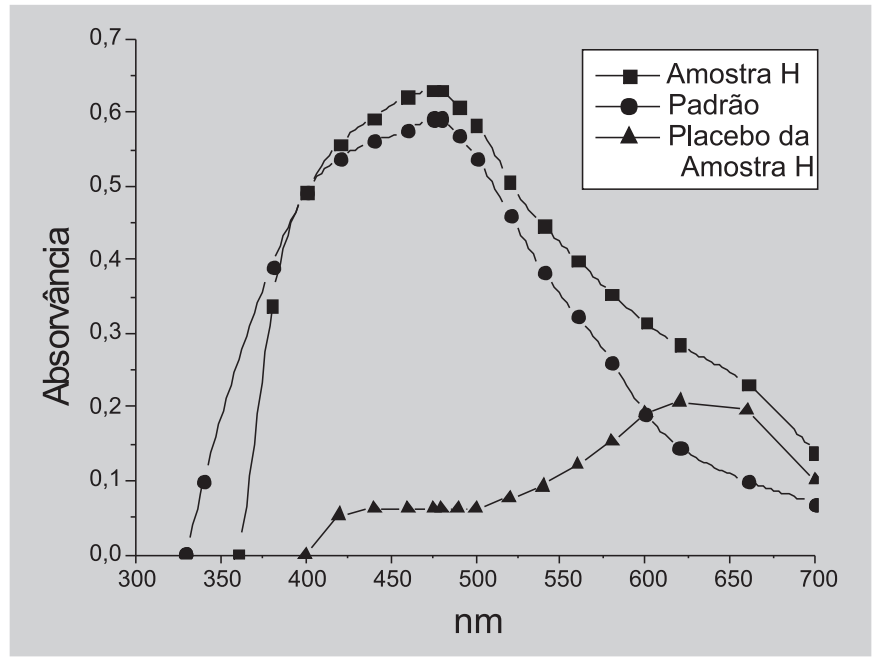

FIGURA 6 - Avaliação da influência do placebo da amostra simulada $\mathrm{H}$ (xarope) no espectro de absorção do produto colorido. Concentração de leitura do padrão e da amostra: 7,0 $\mu \mathrm{g} / \mathrm{mL}$ de BF.

exato e preciso, considerando os valores apresentados, respectivamente, em termos de teor percentual encontrado e coeficiente de variação (inferior a 1\%). Os valores obtidos no teste de recuperação do padrão entre 98,28 e 102,16 \% (Tabela III) confirmam a exatidão do método proposto.

A linearidade é definida como a capacidade que uma metodologia analítica tem de demonstrar que os resultados obtidos são diretamente proporcionais à concentração do analito na amostra em dado intervalo (Brasil, 2003; USP, 2006). Recomenda-se ainda, que sejam analisadas pelo 
TABELA II - Resultados obtidos e tratamento estatístico na determinação do BF em amostras comerciais e simuladas. Leitura a $475 \mathrm{~nm}$

\begin{tabular}{lcccc}
\hline Amostra & $\begin{array}{c}\text { Quantidade experimental } \\
\text { encontrada* }(\mathrm{mg})\end{array}$ & $\begin{array}{c}\text { Teor percentual } \\
\text { encontrado }(\%)\end{array}$ & $\begin{array}{c}\text { Coeficiente de } \\
\text { Variação }(\%)\end{array}$ & $\begin{array}{c}\text { Intervalo de confiança } \\
\text { da média }(\%)\end{array}$ \\
\hline A & 2,433 & 97,32 & 0,56 & $97,32 \pm 0,39$ \\
$\mathrm{~B}$ & 2,495 & 99,82 & 0,54 & $99,82 \pm 0,39$ \\
$\mathrm{C}$ & 1,446 & 96,39 & 0,55 & $96,39 \pm 0,38$ \\
$\mathrm{D}$ & 1,517 & 101,13 & 0,25 & $101,13 \pm 0,18$ \\
$\mathrm{E}$ & 74,490 & 99,32 & 0,62 & $99,32 \pm 0,44$ \\
$\mathrm{~F}$ & 73,627 & 98,17 & 0,58 & $98,17 \pm 0,40$ \\
$\mathrm{G}$ & $* *$ & $* *$ & $* *$ & $* *$ \\
$\mathrm{H}$ & 52,505 & 105,01 & 0,82 & $105,01 \pm 0,62$ \\
\hline
\end{tabular}

*Os valores estão expressos como média de 10 determinações $(n=10)$.

**Os resultados obtidos para a amostra $\mathrm{G}$ não foram considerados porque houve forte interferência dos componentes da fórmula.

TABELA III - Resultados do teste de recuperação do padrão aplicado às amostras comerciais e simuladas

\begin{tabular}{cccc}
\hline Amostra & $\begin{array}{c}\text { Quantidade } \\
\text { de padrão } \\
\text { adicionado } \\
(\mu \mathrm{g} / \mathrm{mL})\end{array}$ & $\begin{array}{c}\text { Quantidade } \\
\text { de padrão } \\
\text { recuperado* } \\
(\mu \mathrm{g} / \mathrm{mL})\end{array}$ & $\begin{array}{c}\text { Percentagem de } \\
\text { recuperação } \\
(\%)\end{array}$ \\
\hline $\mathrm{A}$ & 5,0 & 5,08 & 101,60 \\
$\mathrm{~B}$ & 6,0 & 5,97 & 99,50 \\
& 7,0 & 7,05 & 100,71 \\
$\mathrm{C}$ & 5,0 & 4,99 & 99,80 \\
& 6,0 & 6,09 & 101,50 \\
$\mathrm{D}$ & 7,0 & 7,00 & 100,00 \\
& 5,0 & 4,95 & 99,00 \\
$\mathrm{E}$ & 6,0 & 5,97 & 99,50 \\
& 7,0 & 6,88 & 98,28 \\
& 5,0 & 5,08 & 101,60 \\
$\mathrm{~F}$ & 6,0 & 6,13 & 102,16 \\
& 7,0 & 7,05 & 100,71 \\
& 5,0 & 5,07 & 101,40 \\
& 6,0 & 6,02 & 100,33 \\
& 7,0 & 6,94 & 99,14 \\
& 5,0 & 4,93 & 98,60 \\
& 6,0 & 6,09 & 101,50 \\
& 7,0 & 6,92 & 98,86 \\
\hline
\end{tabular}

*Os resultados estão expressos como média de três determinações $(\mathrm{n}=3)$.

menos cinco concentrações diferentes e que, após a construção gráfica e avaliação estatística dos resultados, o coeficiente de correlação (r) seja no mínimo igual a 0,99. A Figura 7 apresenta os resultados obtidos na análise da linearidade com o placebo da amostra B, confirmando assim que o método proposto é linear. Esta afirmação pode ser referendada após avaliação dos fatores de resposta e obtenção do coeficiente de variação $(\mathrm{CV})$ inferior a $2 \%$, conforme Tabela IV (Leite, 1996).

$\mathrm{Na}$ avaliação da precisão intra-ensaio e inter-ensaio os resultados obtidos, em termos de coeficiente de variação, para a amostra comercial A e amostras simuladas B, D e F, em ambos os experimentos foram de $0,39 \%$ a $0,71 \%$, evidenciando a precisão do método proposto.

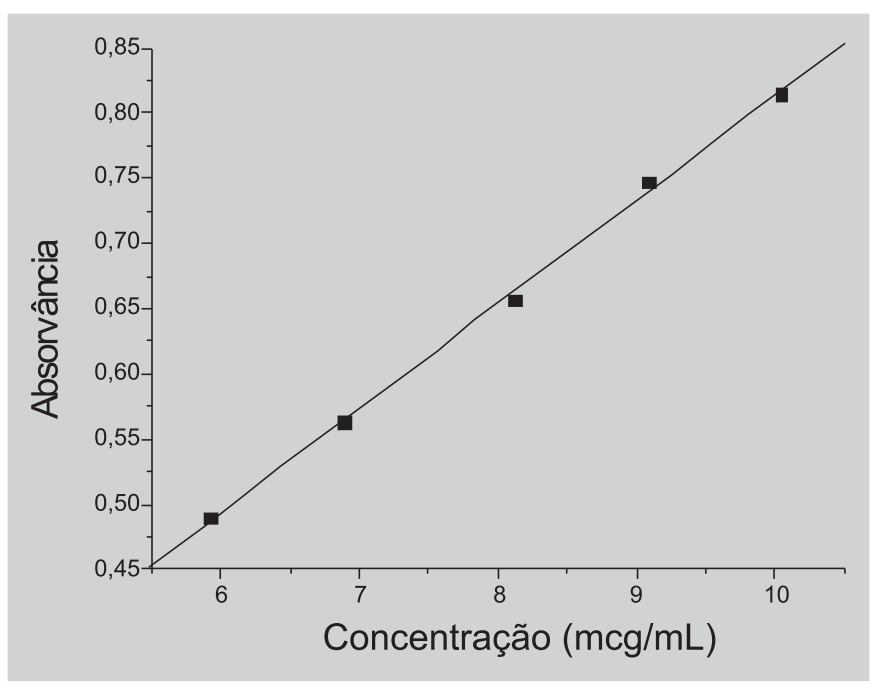

FIGURA 7 - Reta teórica construída para avaliação da linearidade, através do Programa Microcal Origin 6.0, considerando a média das concentrações de bromidrato de fenoterol e das absorvâncias encontradas em três alíquotas, preparadas a partir do placebo da amostra B. As absorvâncias foram determinadas a $475 \mathrm{~nm}$ contra o branco. Valor de $\mathrm{r}=0,99938$. 
TABELA IV - Valores obtidos em termos de fatores de resposta (FR), considerando a concentração de BF e respectivas absorvâncias determinadas em $475 \mathrm{~nm}$, a partir do placebo da amostra $\mathrm{B}$. Os valores expressam a média de três determinações

\begin{tabular}{lccc}
\hline $\begin{array}{l}\text { Concentração } \\
\text { teórica } \\
(\mu \mathrm{g} / \mathrm{mL})\end{array}$ & $\begin{array}{c}\text { Concentração } \\
\text { encontrada } \\
(\mu \mathrm{g} / \mathrm{mL})\end{array}$ & Absorvância & $\begin{array}{c}\text { Fator de } \\
\text { Resposta } \\
(\mathrm{FR})\end{array}$ \\
\hline 6,0 & 5,93 & 0,4895 & 0,08255 \\
7,0 & 6,89 & 0,5633 & 0,08176 \\
8,0 & 8,12 & 0,6571 & 0,08092 \\
9,0 & 9,09 & 0,7471 & 0,08219 \\
10,0 & 10,04 & 0,8144 & 0,08112 \\
\hline
\end{tabular}

FR médio $=0,08171 ; \mathrm{DP}=0,0006912 ; \mathrm{CV}=0,85 \%$.

\section{CONCLUSÃO}

O método espectrofotométrico de quantificação do $\mathrm{BF}$ em gotas e comprimidos, pela reação com o MBTH e sulfato cérico, em meio ácido, mesmo quando associado ao ibuprofeno, mostrou-se preciso, exato, sensível e específico no intervalo de concentração de 3,0 a $12,0 \mu \mathrm{g} / \mathrm{mL}$ de BF.

A curva de calibração foi linear com coeficiente de correlação $(r=0,9998)$ e valores de desvio padrão menores do que $1,0 \%$. Os resultados do teste de recuperação comprovam a exatidão do método e sua aplicação em laboratórios de controle de qualidade de indústrias farmacêuticas, especialmente quando associado ao ibuprofeno, cuja determinação espectrofotométrica no ultravioleta é impossibilitada. O ensaio de linearidade reproduzido com o placebo da amostra B apresentou resultados que são diretamente proporcionais à concentração do analito na amostra, obtendo-se coeficiente de correlação próximo de 1 (um) e coeficiente de variação menor do que $2 \%(\mathrm{CV}=0,85 \%)$, para os fatores de resposta.

O método proposto destaca-se pela facilidade de execução, boa reprodutibilidade e economia, características importantes no controle de qualidade de medicamentos.

Quanto à forma farmacêutica xarope houve interferência do veículo, sugerindo procedimentos prévios de extração ou exploração da espectrofotometria derivada.

\section{ABSTRACT \\ Spectrophotometric determination of fenoterol hydrobromide in pharmaceutical preparations}

A simple spectrophotometric method has been developed for the determination of fenoterol hydrobromide $(\mathrm{FH})$ in tablets, drops and syrup, as the only active principle and associated with ibuprofen. The method is based on the oxidative coupling reaction of the FH with 3-methyl-2benzothiazolinone hydrazone (MBTH) and ceric sulphate as oxidant reagent. The mixture of the drug, MBTH and ceric sulfate, in acid medium, produces a red brown color compound, with absorption maximum at $475 \mathrm{~nm}$. The calibration curve was linear over a concentration range from 3.0 to $12.0 \mu \mathrm{g} / \mathrm{mL}$, with correlation coefficient of 0.9998. The different experimental parameters affecting the development and stability of the color compound were carefully studied and optimized. The method was applied successfully to assay $\mathrm{FH}$ in dosage forms and simulated samples. The coefficient of variation was from $0.25 \%$ to $0.82 \%$ and average recoveries of the standard from $98 \%$ to $102 \%$. The excipients (tablets and drops) did not interfere in the analysis and the results showed that method can be used for determination of the FH isolated or associated with ibuprofen with precision, accuracy and specificity. In case of syrup, the interference in the analysis suggests a possible reaction between vehicle components with MBTH.

UNITERMS: Fenoterol hydrobromide/determination. 3-methyl-2-benzothiazolinone hydrazone. Pharmaceutical forms/analysis. Spectrophotometry/drug's analysis.

\section{REFERÊNCIAS BIBLIOGRÁFICAS}

BELAL, F.; AL-MALAQ, H.A.; AL-MAJED, A.A. Voltammetric determination of isoxsuprine and fenoterol in dosage forms and biological fluids through nitrosation. J. Pharm. Biomed. Anal., v.23, p.1005-1015, 2000.

BEYENE, N.W.; STADEN, J.F.; STEFAN, R.I. Determination of fenoterol hydrobromide by sequential injection analysis with spectrophotometric detection. Anal. Chim. Acta, v.521, p.223-229, 2004.

BRASIL. Resolução RE nº 899, de 29 de maio de 2003. Determina a publicação do "Guia para validação de métodos analíticos e bioanalíticos". Disponível em: $<$ http://e-legis.anvisa.gov.br/leisref/public/ showAct.php?id=15132\&word $=$ Guia $>$. Acesso em: 1 maio 2007.

BRITISH Pharmacopoeia 2007. London: Her Majesty's Stationery Office, 2007. p.671. 
DAMASCENO, L.; VENTURA, R.; CARDOSO, J.; SEGURA, J. Diagnostic evidence for the presence of bagonists using two consecutive derivatization procedures and gás chromatography-mass spectrometric analysis. $J$. Chromatogr., B, v.780, p.61-71, 2002.

EL-EMAM, A.A.; BELAL, F.F.; MOUSTAFA, M.A.; ELASHRY, S.M.; EL-SHERBINY, D.T.; HANSEN, S.H. Spectrophotometric determination of propranolol in formulations via oxidative coupling with 3methylbenzothiazoline-2-one hydrazone. Farmaco, v.58, p.1179-1186, 2003.

EL-SHABRAWY, Y.; BELAL, F.; EL-DIN, M.S.; SHALAN, S. Spectrophotometric determination of fenoterol hydrobromide in pure form and dosage forms. Farmaco, v.58, p.1033-1038, 2003.

GARFIELD, F.M. Quality assurance principles for analytical laboratories. Arlington: Association of Official Analytical Chemistry International, 1994. p.74-81.

GEETA, N.; BAGGI, T.R. Rapid spectrophotometric determination of aspirin using 3-methylbenzthiazolinone2-hydrazone. Microchem. J., v.38, p.232-235, 1988.

JACOBSON, G.A.; PETERSON, G.M. High-performance liquid chromatographic assay for the simultaneous determination of ipratropium bromide, fenoterol, salbutamol and terbutaline in nebulizer solution.J. Pharm. Biomed. Anal., v.12, n.6, p.825-832, 1994.

KRAMER, S.; BLASCHKE, G. High-performance liquid chromatographic determination of the $\beta_{2}$-selective adrenergic agonist fenoterol in human plasma after fluorescence derivatization. J. Chromatogr., B, v.751, p.169-175, 2001.

LEITE, F. Validação em análise química. Campinas: Átomo, 1996. $120 \mathrm{p}$.
MEINEKE, I.; STEINMETZ, H.; KRAMER, S.; GLEITER, C.H. Determination of fenoterol in human plasma by HPLC with fluorescence detection after derivatization. $J$. Pharm. Biomed. Anal., v.29, p.147-152, 2002.

NAGARAJA, P.; SUNITHA, K.R.; VASANTHA, R.A.; YATHIRAJAN, H.S. Spectrophotometric determination of metronidazole and tinidazole in pharmaceutical preparations. J. Pharm. Biomed. Anal., v.28, p.527-535, 2002.

NIKOLIC, K.; ARSENIJEVEC, L.; BOGAVAC, M. Coulometric determination of some antiasthmatics. $J$. Pharm. Biomed. Anal., v.11, p.207-210, 1993.

PRASAD, A.V.S.S.; LAKSHMI, C.S.R.; SASTRY, C.S.P.; UPPULETI, V.P. Determination of minocycline by oxidative coupling and diazocoupling reactions in pharmaceutical formulations. J. Pharm. Biomed. Anal., v.30, p.491-498, 2002.

RAHMAN, N.; KHAN, N.A.; AZMI,S.N.H. Optimized and validated spectrophotometric methods for the determination of nicorandil in drug formulations and biological fluids. Farmaco, v.59, p.519-527, 2004.

RAO, G.R.; AVADHANULU, A.B.; GIRIDHAR, R.; KOKATE, C.K. Spectrophotometric determination of guaiphenesin in formulations using 3-methyl-2benzothiazolinone hydrazone hydrochloride. East. Pharm., v.31, n.367, p.141-142, 1988.

SULTAN, M. Spectrophotometric determination of acyclovir in some pharmaceutical formulations. Farmaco, v.57, p.865-870, 2002.

UNITED States Pharmacopeia. 29.ed. Rockville: United States Pharmacopeial Convention, 2006. p.3050-3052.

VOGEL, A.I. Análise química quantitativa. 6.ed. Rio de Janeiro: Livros Técnicos Científicos, 2002. p.353-385.

Recebido para publicação em 19 de junho de 2007 Aceito para publicação em 30 de julho de 2008 